\title{
Shoulder isokinetic exploration following shoulder rotator cuff suture
}

\author{
J. Binet ${ }^{\mathrm{a}}$, B. Forthomme ${ }^{\mathrm{b}}$, J.P. Bierlaire ${ }^{\mathrm{a}}$, P. Meurant ${ }^{\mathrm{a}}$, J.M. Crielaard ${ }^{\mathrm{b}}$ and J.L. Croisier $^{\mathrm{b}}$ \\ ${ }^{a}$ Department of Physical Medicine and Rehabilitation, CHR, Tournai, Belgium \\ ${ }^{\mathrm{b}}$ Department of Physical Medicine and Rehabilitation, CHU Sart Tilman, Li ège, Belgium
}

The rotator cuff lesion is the most common shoulder pathology. After a well-achieved rehabilitation program of a few months, our patients' mobility recovery is most of the time complete. The goal of this study is to evaluate the muscle strength at the end of the rehabilitation.

The follow-up deals with 42 cases operated by two surgeons. Three surgical techniques were used: simple suture (3 cases), cuff-to-bone reinsertion (5 cases) and anchoring technique (34 cases). Six to eight months after the operation, the patients underwent a standard clinical examination and an isokinetic exploration (Cybex Norm) measuring the internal and external rotators and the abductors-adductors peak torques. As far as the rotators are concerned, this occurred according to the following criteria: patient lying supine, arm abducted to 90 degrees in the frontal plane (the engine rotating axis matches with the gleno-humeral joint centre). Three speeds were taken into account: 60,120 and 240 degrees per second respectively repeated 3, 4 and 5 times (rest period between each set is 1 minute). As far as the abductors-adductors are concerned, this occurred according to the following criteria: patient lying on his/her side, dynamometer behind the patient, against rest put on the distal part of the arm, amplitude purposely ranging from 0 to maximum 90 degrees of abduction. Two speeds were taken into account: 60 and 180 degrees per second respectively repeated 3 and 4 times. Student's T-test and correlation studies were carried out to statistically analyze the range of motion, the peak torques (absolute and relative values) and the ratios of the joint.
From the point of view of strength when comparing at low speed to the non-operated arm, men showed a deficiency as high as $16 \%$ for the external rotators (Fig. 1), 6\% for the internal rotators, $21 \%$ for the abductors (Fig. 2) and 7\% for the adductors. Women's deficiency turned out to reach $21 \%$ for the external rotators (Fig. 1), 5\% for the internal rotators, $23 \%$ for the abductors (Fig. 2) and $10 \%$ for the adductors.

Dominant-side is of paramount importance in strength recovery. The biggest difference is to be noticed on the internal rotators showing an $18 \%$ deficiency in patients operated on the non-dominant side whereas the dominant-side operated patients did not show any deficiency.

Comparing dominant-side operated to non-operated sedentary men showed a weakening of $15 \%$ for the external rotators (very significant) at low speed affecting the ratio and diminishing of the abductor-adductor torque of $26 \%$ for the abductors and $20 \%$ for the adductors. When considering female patients, the same comparison at low speed showed a deficiency as high as $34 \%$ for the external rotators, $28 \%$ for the abductors and $36 \%$ for the adductors.

In the group of non-dominant side operated patients, we found a correlation between external rotators deficiency at a speed of 240 degrees per second and preoperative pain for more than six months.

Male patients showed a full recovery of the range of motion and female patients showed an external rotation deficiency as high as 5\% (significant) whereas the internal rotation deficiency reached $10 \%$ (very significant). 


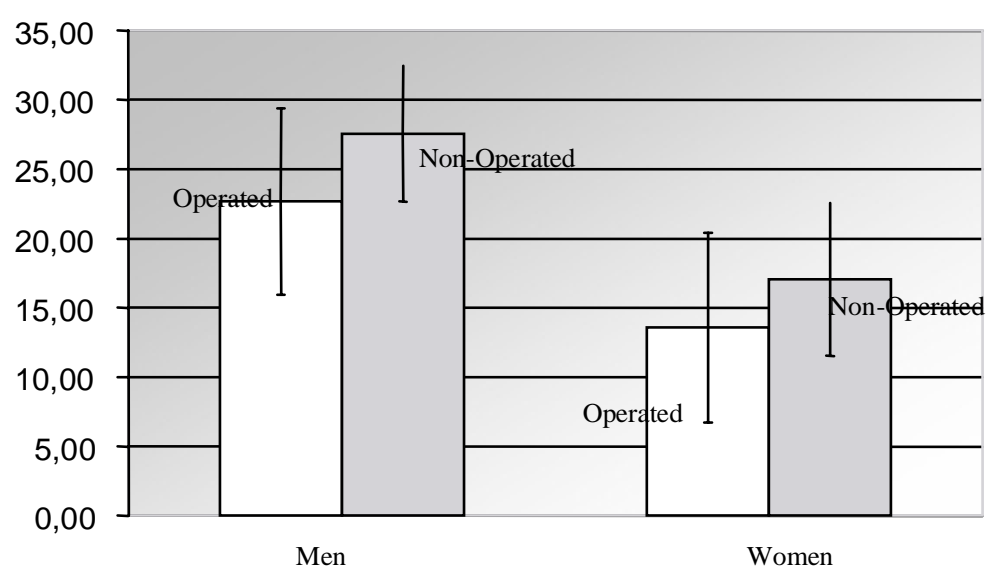

Fig. 1. Shoulder External rotator peak torques (in N.m) comparison at $60 \% / \mathrm{sec}$ between operated and non-operated arms of men and women.

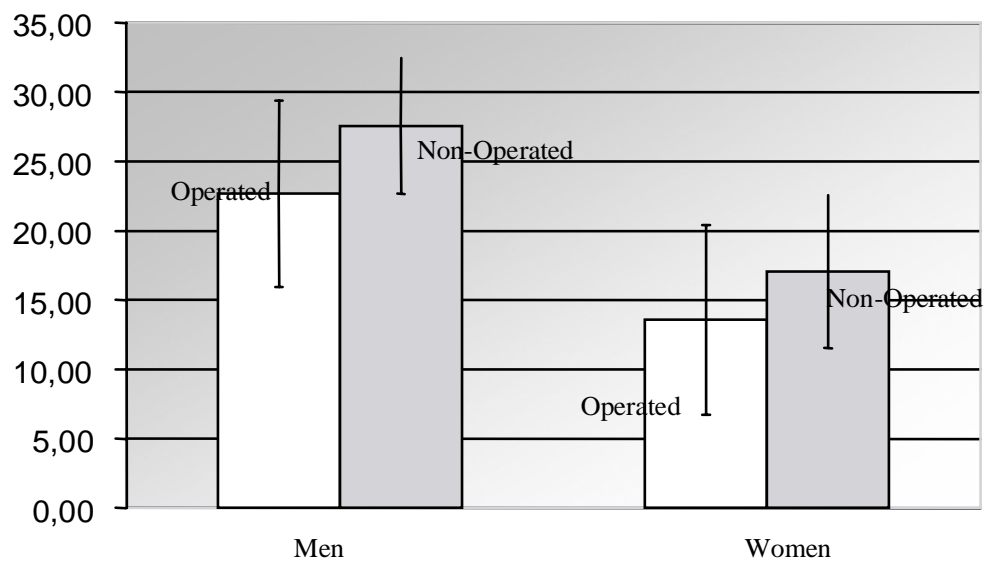

Fig. 2. Shoulder abductor peak torques (in N.m) comparison at $60^{\circ} / \mathrm{sec}$ between operated and non-operated arms of men and women.

In spite of discrepancies from a statistical point of view, it is to be noticed that men and women complied with a similar strength recovery profile featuring a maintained deficiency at external rotator and abductor level. Total mobility could easily be achieved with men whereas women seemed to have some trouble with the internal rotation recovery.

Consequently, our patients did still show abnormalities six months later, which made us wonder whether the rehabilitation was not ended too prematurely. 